\title{
Form Finding of Shell Bridges Using the Pneumatic Forming of Hardened Concrete Construction Principle
}

\author{
Benjamin Kromoser $\mathbb{D}^{1},{ }^{1}$ Thomas Pachner, ${ }^{2}$ Chengcheng Tang, ${ }^{3}$ Johann Kollegger, ${ }^{4}$ \\ and Helmut Pottmann ${ }^{5}$ \\ ${ }^{1}$ Institute of Structural Engineering, University of Natural Resources and Life Sciences Vienna, Peter-Jordan-Straße 82, \\ 1190 Vienna, Austria \\ ${ }^{2}$ Patonic, Trattnachtalstraße 1a, 4710 Grieskirchen, Austria \\ ${ }^{3}$ Computer Science Department, Stanford University, Stanford, CA 94305, USA \\ ${ }^{4}$ Institute for Structural Engineering, TU Wien, Karlsplatz 13, E212-2, 1040 Vienna, Austria \\ ${ }^{5}$ Center for Geometry and Computational Design, TU Wien, Wiedner Hauptstraße 8-10/104, 1040 Vienna, Austria
}

Correspondence should be addressed to Benjamin Kromoser; benjamin.kromoser@boku.ac.at

Received 7 September 2018; Accepted 9 October 2018; Published 29 November 2018

Academic Editor: Melina Bosco

Copyright (c) 2018 Benjamin Kromoser et al. This is an open access article distributed under the Creative Commons Attribution License, which permits unrestricted use, distribution, and reproduction in any medium, provided the original work is properly cited.

\begin{abstract}
Concrete shells are fascinating structures. Even thin shells can span over large areas without requiring any columns. If a formdefining load case exists, the shape of the shell can be designed to ensure that the forces in the structure are transferred primarily by the membrane action, which leads to an even distribution of the stresses across the shell surface. Concrete as a material, characterized by high compressive strength and low tensile strength, can be used with a very high degree of utilization. A fundamental problem with building concrete shells is the high effort required for the production of the complicated formwork. A new construction principle called Pneumatic Forming of Hardened Concrete (PFHC) was invented at TU Wien and requires no traditional formwork or falsework during the construction process. An air cushion is used to lift a flat hardened concrete plate, and at the same time, additional post-tensioning cables are tightened to support the transformation of the flat plate into a doublecurved shell. One possible application of PFHC is the construction of shell bridges. Here, the shape of the shell has to be designed according to the acting loads and the boundary conditions of the construction method. This paper describes the partly conflicting factors involved in the form-finding process for practical application and the semiautomated workflow for optimizing the geometry of shell bridges. In the first optimization step, the final bridge shape is determined using a particle-spring system or alternatively a thrust-network approach. In the second optimization step, the shell is completed to form a full dome-this is called the reference geometry and is required for the new construction method. Finally, the reference geometry is discretized into singlecurved panels by using a mesh-based optimization framework. To frame the presented work, an overview of different experimental and computer-aided form-finding methods is given.
\end{abstract}

\section{Introduction}

Concrete has a high compressive strength and a low tensile strength. Unfortunately, however, in the design of most concrete structures, this fact is not considered. High tensile stresses, which are found in most structures and are caused by bending moments, shear forces, and torsional moments, lead to low utilization of the material and require a large amount of additional reinforcement. By contrast, in correctly designed concrete shells, the loads are mainly transferred by compressive forces. This leads to high utilization of the material and reduces the amount of required reinforcement.

Even so, few concrete shells are built today. The main reason for this is that the construction of the complicated formwork and falsework is material and labor intensive, and these auxiliary structures can only be used once in most of the cases. 
It is possible to significantly reduce the effort required for the production of the complicated formwork and falsework using a pneumatic formwork. As described in [1], many different pneumatic formwork methods exist. However, many of these systems are form-active, meaning that the shape of the pneumatic formwork mirrors the shape of the finished structure. This leads to process-related inaccuracies with respect to the reference geometry and the shell thickness, resulting in deviations from the planned structure. Because of these disadvantages, a new construction principle using a pneumatic air cushion in a different way was invented at TU Wien, as described in [2-5].

However, designing a concrete shell bridge under consideration of the relevant loads and the boundary conditions of the selected construction method is a very challenging task which will be addressed in this paper.

After an introduction to the topic, an overview of different form-finding methods for concrete shells is given in Section 2. In Section 3, the functionality of PFHC is described. The methodology used for applying the method to shell bridges is explained in Section 4. Section 5 deals with the different boundary conditions, and in Section 6, the complete workflow for the design process of shell bridges built with the PFHC method as well as the computational geometry optimization process is presented. Furthermore, a new approach for determining the reference geometry using an optimization algorithm based on a thrust network is proposed. The results and proposed further research are discussed in Section 7.

\section{Overview of Form-Finding Methods for Concrete Shells}

2.1. Fundamentals for Form Finding of Concrete Shells. As mentioned in [6], a form-defining load case is crucial in determining the optimum shape of a structural element. Assuming that a form-defining load case exists, concrete shells can be designed according to the acting loads. In a concrete shell used as deer overpass, for example, the formdefining load case consists of the structure self-weight and the earth loads. The main objective is to determine a layout and shape that minimizes the bending moments and maximizes the stiffness of the shell. This can generally be achieved by minimizing the strain energy $S_{\mathrm{E}}$ [7]. $S_{\mathrm{E}}$ can be defined as follows:

$$
S_{\mathrm{E}}=\frac{1}{2} \int \sigma \varepsilon d V
$$

where $\sigma$ is the stress and $\varepsilon$ is the strain. Both stresses and strains are calculated for each point of the shell.

Physical models or their simulation equivalents offer the opportunity to determine surfaces with minimal strain energy [8]. Soap-film models represent the equilibrium state with the lowest strain energy for shells with negative Gaussian curvature and hanging models for shells with a positive Gaussian curvature. To also achieve minimization of the displacement, a volumetric-displacement function can be defined according to [9]:

$$
\mathrm{VD}=\sum_{i=1}^{n} d_{i} S_{i} \text { Thick }_{i}
$$

where $d_{i}$ is the displacement vector modulus at each point $i$, $S_{i}$ denotes the area of influence at point $i$, and Thick $_{1}$ is the average thickness of area $S_{i}$.

At the start of the optimization, it is crucial to determine an objective function. This could, for example, be the weight, the strain energy $S_{\mathrm{E}}$, or the stress leveling. The stress leveling $S_{\mathrm{L}}$ can be defined as follows:

$$
S_{\mathrm{L}}=\sum_{i j}\left(\sigma_{i j}-\sigma_{a}\right)^{2}, \quad i=1,2,3, \ldots, j=1,2, \ldots, n .
$$

It is also possible and useful to implement further influencing factors with appropriate weighting within the optimization so as to achieve application-specific improvements in the structural behavior of the shell. Areas of improvements include the buckling load, the buckling behavior, and the resistance to unexpected asymmetric loads.

However, the requirements of different shell structures diverge, and the optimization criteria have to be adapted according to these requirements. Thus, it falls to the engineers carrying out the optimizations to define a customized optimum shape for the designed structure.

2.2. Experimental Form-Finding Methods. Despite continuous improvements in numerical form-finding methods, the traditional experimental form-finding methods naturally form the fundamental basis for any improvements. For instance, Frei Otto used soap-film models to define the shapes for his hanging structures [10]. Antoni Gaudí defined the form of buildings by using hanging chain models, and Isler used hanging membranes to define the shape of shells [11]. By adding more weights to the physical models, their shapes can be adjusted to support additional external loads besides the self-weight of the shell. The determined form results in a uniform stress distribution in the undeformed structure. Isler also tested another experimental formfinding method which uses inflated membranes. As described in [6], unfortunately, the internal pressure of the membranes generates a false load case, since there is no load acting opposite to the internal pressure after deflation of the membrane. Thus, the stresses in the fabricated shell deviate from the ideal equalized membrane state of stress due to selfweight, particularly in flat shells. Consequently, inflated membranes are not ideal as physical form-finding methods.

2.3. Computational Form-Finding Methods. Since the 1960s, numerical form-finding methods have been improving continuously. Various approaches by different researchers have led to a large variety of solution strategies. The properties of the soap-film models, for example, were implied in methods such as the dynamic relaxation method [10], the force density method [12], the updated reference strategy (URS) [13], and methods of modified linearization $[14,15]$. Since a comparison between the different methods is difficult, Veenendaal and Block [16] give an overview of 
these structural form-finding methods and compare them by analyzing their advantages and disadvantages in detail.

To define the shape of a concrete shell with positive Gaussian curvature in which the relevant loads are transmitted predominantly by in-plane compressive forces, hanging models that are described by conventional nonlinear finite-element analysis of elastic bodies with additional boundary conditions can be used. A structure that is unable to resist bending or shear, such as an elastic net structure, serves as the logical basis.

In contrast to experimental form-finding methods, correctly programmed numerical methods allow for a more precise determination of the optimum shell shape and consideration of a wider range of boundary conditions. For example, numerical methods allow the implementation of infinitely variable vertical loads, such as self-weight, snow, vertical earth load, or live loads, as well as horizontal loads, such as horizontal earth pressure or water pressure. Thus, numerical methods represent a powerful further development of the existing experimental methods, and completely new solutions of higher complexity can be found.

\section{The Pneumatic Forming of Hardened Concrete Construction Principle}

The PFHC construction method represents a new way for building double-curved shells economically and in a laborand resource-efficient manner. As described in [2-4], a flathardened concrete plate is transformed into a double-curved shell with the aid of an air cushion and post-tensioning tendons. During the transformation process, the plate is lifted and distorted by inflating the air cushion and tensioning the post-tensioning tendons as shown in Figure 1. Pneumatic formworks can be divided into three different categories: (1) air-space pneumatic formworks, (2) classical pneumatic formworks, and (3) lifting pneumatic formworks [1]. Air-space pneumatic formworks are placed inside a conventional formwork to leave a space in the concrete structure. Classical pneumatic formworks are inflated, and the concrete-most commonly sprayed concrete-is applied subsequently. Lifting pneumatic formworks serve as lifting devices for transforming a structure by inflation. They can be divided into two further subcategories: (1) form-active lifting pneumatic formworks and (2) active-bending lifting pneumatic formworks. In contrast to all other pneumatic formworks, active-bending lifting pneumatic formworks do not influence the final form of the structure, and imperfections due to the construction method are reduced. The presented construction method, PFHC, belongs to the category of active-bending lifting pneumatic formworks. The membrane serves merely as a lifting device and only slightly influences the final form of the shell.

Erecting a shell from a flat plate induces large strains in the circumferential direction. To absorb these strains, wedge-shaped gaps are formed in the flat concrete plate, using a single-curved formwork. An air cushion with a only slightly smaller diameter to that of the initially flat concrete plate is used to lift the structure and transform it into a double-curved shell. A thicker plate edge prevents the whole plate from lifting off the ground. The flat plate consists of a middle part and several wedge-shaped elements which are monolithically connected to the middle part (Figure 1). Pneumatic wedges are placed between the concrete elements to protect the pneumatic formwork in the area of the wedgeshaped gaps during the erection process. Using reinforcement with linear-elastic material behavior, a low modulus of elasticity $\left(50,000-100,000 \mathrm{~N} / \mathrm{mm}^{2}\right)$ and high ultimate strain $(>2 \%)$, e.g., steel ropes or glass-fiberreinforced plastic rods, allow for large strains in the concrete members. Detailed results of different preliminary bending tests on shells made of $0.1 \mathrm{~m}$ and $0.12 \mathrm{~m}$ thick plates are described in [5]. As explained in [3], the newly invented construction method could be used for a range of doublecurved surfaces with a positive Gaussian curvature. Because concrete is a brittle material, concrete plates cannot absorb large strains in the plane of the plates, but they can be bent uniaxially. Hence, developable surfaces have to be used. The most challenging aspect of the design of these surfaces is the production of nonoverlapping representations consisting of developable single-curved strips [17].

\section{Construction of a Shell Bridge Used as a Deer Overpass}

As described in [3-5], one possible application of the new construction method is the construction of shell bridges. A shell bridge with the plan dimensions (without considering the foundations) of $36.2 \times 38.7 \mathrm{~m}$, a final height of $8.1 \mathrm{~m}$, and a thickness of $0.45 \mathrm{~m}$, which was built with PFHC in Carinthia in Austria, is shown in Figure 2. A thin (for example, $50-150 \mathrm{~mm}$ ), initially flat reinforced concrete plate is transformed into a double-curved shell consisting of singlecurved panels. This structure serves as a kind of the lost formwork and will eventually be part of the final supporting structure. After the surface has been roughened in the relevant areas by high-pressure water jetting, the required reinforcement layers and additional concrete layers are placed onto the thin shell in the area of the final bridge, as shown in Figure 3. Accompanying additional reinforcement is drilled into the foundations to create a connection between shell and foundation. In the next step, an additional, thicker concrete layer is applied in situ (sprayed concrete or a conventionally poured concrete top layer). Finally, the temporarily used tendons and the concrete are removed in the areas of the bridge portals, and the edge beam is cast. The removed parts represent fewer than $2 \%$ of the complete concrete mass of the bridge as the removed parts have only the small thickness of the inflated shell (without additional added concrete). Any small cracks caused by the transformation process are filled during the additional concrete layer is added, and the shell including the lost formwork and the additional concrete layer acts as a monolithic structure. If more than one layer of reinforcement is required and sprayed concrete is used, the additional concrete layer must be applied in more than one work step to ensure good bonding properties. Exemplarily all work steps for the construction of a shell bridge built with PFHC are shown in Figure 3. In comparison to a conventional frame bridge, the 

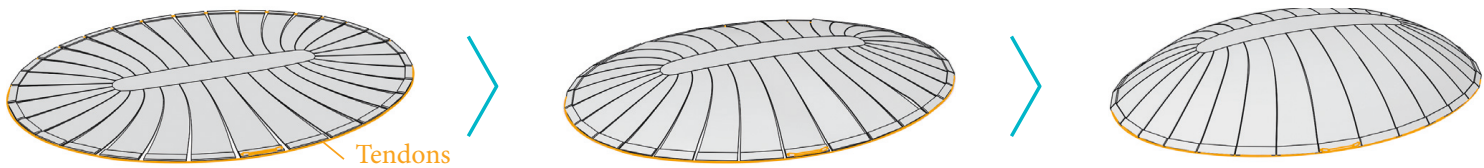

Figure 1: Construction principle of the Pneumatic Forming of Hardened Concrete (PFHC) construction method.

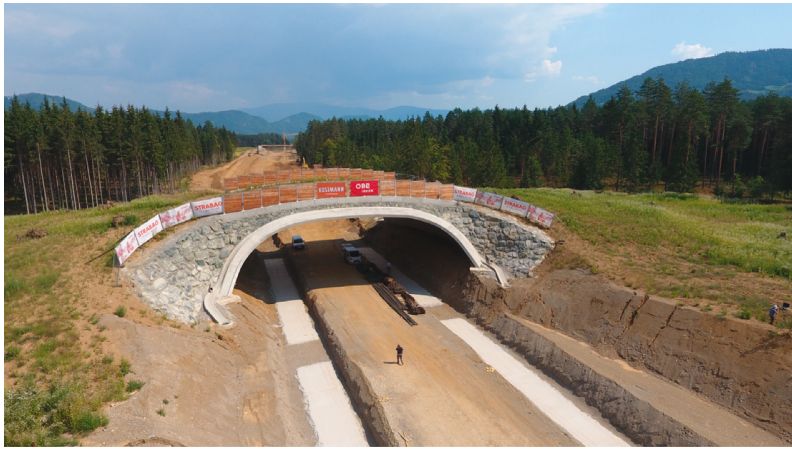

Figure 2: Shell bridge built in Carinthia in Austria with PFHC (plan dimensions: $36.2 \times 38.7 \mathrm{~m}$, height: $8.1 \mathrm{~m}$, and thickness: $0.45 \mathrm{~m}$ ).

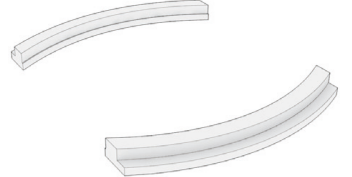

(1)

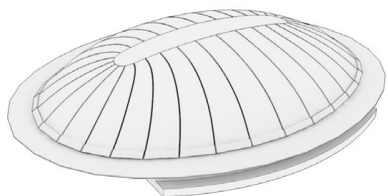

(4)

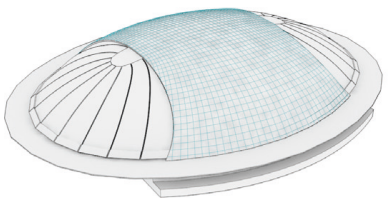

(7)

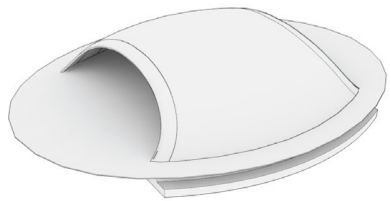

(10)
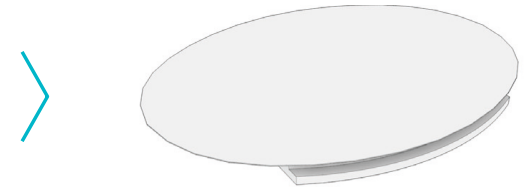

(2)
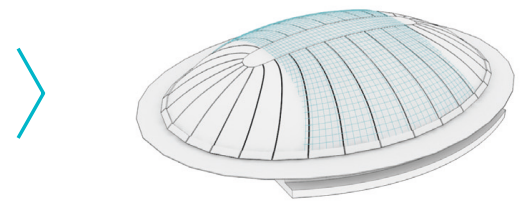

(5)
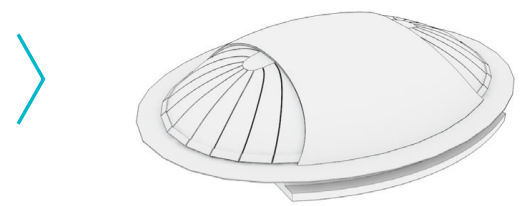

(8)

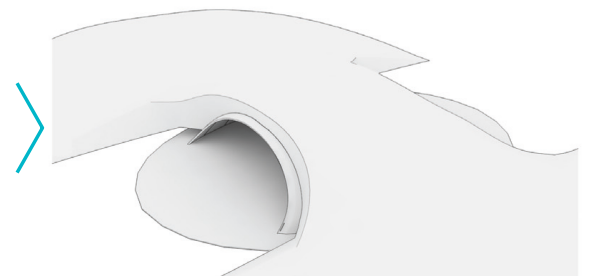

(11)

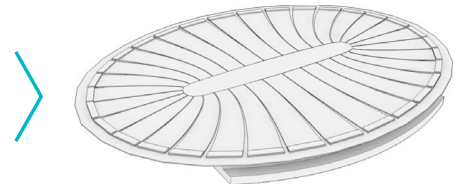

(3)

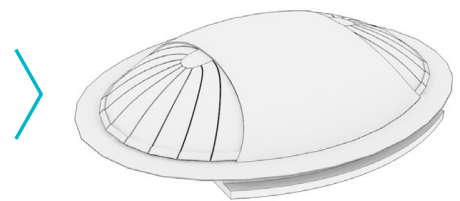

(6)

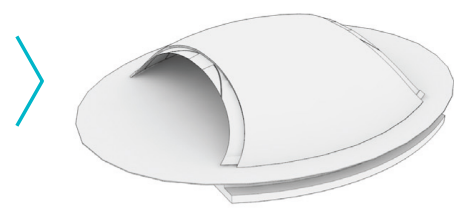

(9)

FIgURE 3: Work steps for the construction of the deer overpass: (1) manufacturing of the foundations; (2) production of the flat working surface out of concrete (the tension plate for the building condition); (3) concreting of the flat concrete plate for the transformation process (thickness $100 \mathrm{~m}$, weight $546 \mathrm{t}$ ); (4) transformation of the initially flat hardened concrete plate to the designed shell; (5)-(8) production of the additional reinforced concrete in layers (total thickness $350 \mathrm{~mm}$ ); (9) cutting out of the portals; (10) production of the edge beam; (11) production of the earth back filling [18]. 
costs can be reduced by approximately $20 \%$ and the $\mathrm{CO}_{2}$ equivalents can be reduced by approximately $40 \%$ [18] due to the fewer required reinforcement. By using the PFHC, the construction principle for the shell bridge shown in Figure 2 allows for a cost reduction of approximately $30 \%$ in comparison to the same shell bridge built with a conventional double-curved formwork and framework.

\section{Boundary Conditions for Designing a Shell Bridge with the PFHC Construction Principle}

The form-finding process for shell bridges built with PFHC is driven by various, sometimes conflicting, factors arising from three main categories of requirements. In order to fulfill the decisive ecological and economic requirements, the material consumption has to be minimized. However, certain minimum dimensions need to be maintained to ensure the usability of the bridge. Furthermore, various criteria have to be fulfilled to guarantee the feasibility of using PFHC. Figure 4 gives a detailed overview of the different requirements.

\section{Geometry Optimization}

Because of the range of existing requirements for a bridge and the production-process-related requirements, the optimization process has been split into four steps as shown in Figure 5. First, the reference geometry of the final bridge is determined. Secondly, two surface patches are added to complete the reference geometry to form a full cupola. Thirdly, the resulting geometry, consisting of a nonuniform rational B-spline surface (NURBS), undergoes a discretization process, resulting in semidiscrete segments that are subsequently unrolled. The optimization steps are described in detail in the following subsections.

6.1. Numerical Form Finding of the Reference Geometry by Using a Particle-Spring System (Step 1). The shape of the reference geometry is mainly affected by three factors: the geometric optimization with respect to the acting loads, the minimization of the required construction material, and the procedural requirements from the PFHC construction method. Thus, a design tool based on a dynamic-equilibrium method [16] was developed to allow a precise and objectspecific determination of the optimized shape. The approach chosen in this paper was to develop a process in which an automated global optimization algorithm is combined with local modifiers that allow manual adjustments of the shell geometry, thereby fulfilling the procedural requirements. This allows a fine adjustment of the final form by the designing engineers.

6.1.1. Form-Finding Method. Within the environment of a dynamic particle-spring system, a base mesh [19], resembling the footprint of the final shell, is constructed as shown in Figure 6. Each nodal point of the mesh represents a particle. One or more forces transform the geometry of the elastic mesh into its final form. The deformed mesh geometry is a result of the equilibrium state of the particles due to the acting forces. This state is calculated using a physics-based geometric modeling approach realized with Kangaroo [20], the CAD environment Rhinoceros [21], and its plugin Grasshopper [22]. Figure 7 shows the forces acting on one particle of the mesh.

6.1.2. Global Optimization Algorithm. In the first step, Hooke's law spring function that simulates cohesion forces among adjacent nodal points is applied to the mesh (Figure 8). These forces cause the particles to act as one system. If a force is applied at one point, the whole system is affected. The intensity of the attractive or repulsive force between each pair of nodes can be modified by the rest length of the spring, a factor that can be used to determine their optimum distance.

The nodal points that represent the base line of the bridge were kept fixed as shown in Figure 9.

The optimum shape of a concrete shell structure has been found when the acting loads induce predominantly evenly distributed compressive normal forces. The defined constraints for this optimization were invariable load, equal thickness, and equal material properties across the entire shell. The approach of the algorithm is to simulate loads at each nodal point of the shell by applying forces acting in the opposite direction. Figure 10 shows the forces applied for shell self-weight, vertical earth load (orange), and horizontal earth pressure (blue).

The optimum geometry, in which the structure experiences mainly compressive normal forces, can be found by combining the spring function that simulates cohesion with the forces acting in the opposite direction to the loads.

6.1.3. Minimal Enclosure of the Structural Gauge. Figure 11 shows the minimum structural gauge of the railway track that needs to be guaranteed. The distance between the foundations (a) has to harmonize with the rise of the foundation arc (b) and the vertical forces acting on the mesh representing the real vertical forces in the opposite direction (c). Choosing an extruded arc as the design surface $(b=0)$ would require a minimum amount of material. However, a foundation arc with $b>0$ is necessary to reduce the horizontal forces by activating a biaxial load-bearing behavior, to meet the requirements of the soil conditions and to ensure better applicability of PFHC.

6.1.4. Low Curvature at the Edges. As explained in [3], a higher edge thickness of the flat initial concrete plate is required to support the bending process from a flat plate to the double-curved shell. A plate of constant thickness without any additional weight at its edge would not distort and instead be lifted as a whole. Due to the thicker edge, this outer part remains undistorted during the transformation. This procedural requirement is taken into account with the help of a local modifier that determines the variation in the magnitude of the vertical forces and thus prevents the edges 


\begin{tabular}{|c|c|c|}
\hline Usability & Economic & Procedural \\
\hline $\begin{array}{l}\text { Minimal gauge for } \\
\text { subjacent trail }\end{array}$ & $\begin{array}{l}\text { Minimized cupola } \\
\text { surface }\end{array}$ & $\begin{array}{c}\text { Positive gaussian curvature } \\
\text { of reference geometry }\end{array}$ \\
\hline \multirow[t]{3}{*}{$\begin{array}{l}\text { Minimum with at } \\
\text { shell vertex }\end{array}$} & \multirow{3}{*}{$\begin{array}{c}\text { Overall reduction of } \\
\text { quantities (concrete, } \\
\text { steel, etc.) }\end{array}$} & $\begin{array}{l}\text { Complete cupola for lifting } \\
\text { process }\end{array}$ \\
\hline & & $\begin{array}{c}\text { Concrete segments can only } \\
\text { be bent uniaxial }\end{array}$ \\
\hline & & $\begin{array}{l}\text { Little segment-bending at } \\
\text { foundation interface }\end{array}$ \\
\hline
\end{tabular}

FIgURE 4: Classification of requirements.
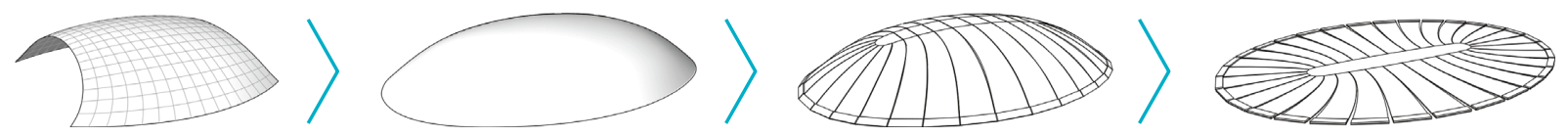

FIGURE 5: Methodology of the optimization process, starting with the optimization of the shape of the final bridge and ending with the initial flat concrete plate required for PFHC.

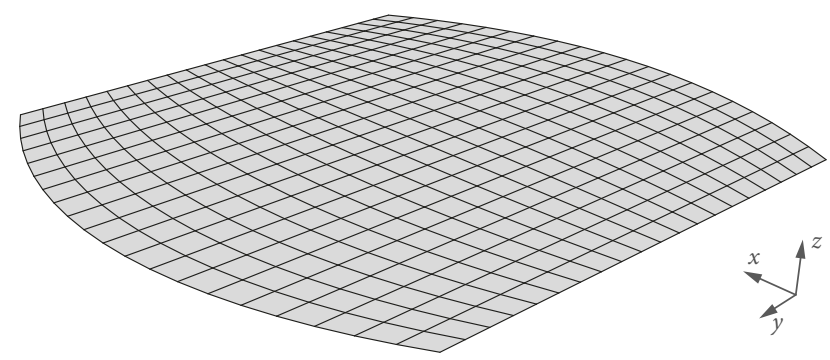

Figure 6: Base mesh resembling the shell footprint, prior to deformation by virtual forces acting in the reverse order to the real loads.

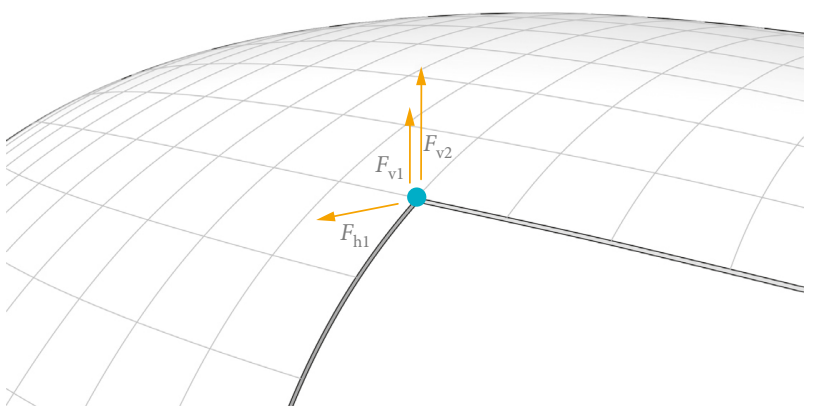

FIGURE 7: Forces acting on one particle of the mesh [23].

of the concrete plate from bending. An evaluation was carried out using a curvature graph as shown in Figure 12.

6.1.5. Minimum Width at the Vertex of the Bridge. The attracting forces between the particles are simulated using Hooke's spring function. As explained above, their intensity can be modified by changing the rest length. To adapt the width of the vertex to the regulatory requirements of the bridge and to improve the structural behavior, the cohesion forces in

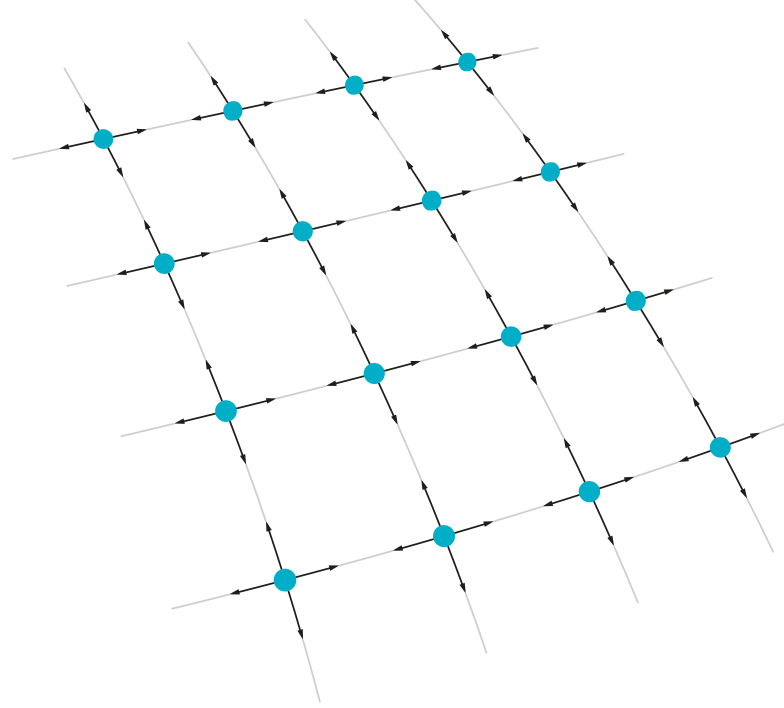

Figure 8: Principle of Hooke's law spring function.

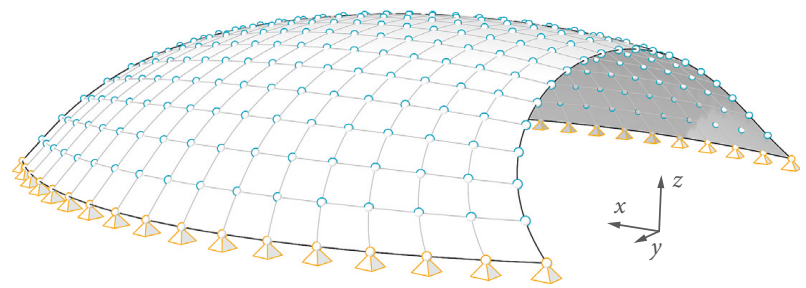

Figure 9: Fixed nodes at the foundations.

the transverse direction are decreased, while the forces in the longitudinal direction are kept constant. The resulting deformation of the geometry is shown in Figures 13 and 14.

Using numerical stress analysis, it was shown that the earth pressure, which varies according to location and is 


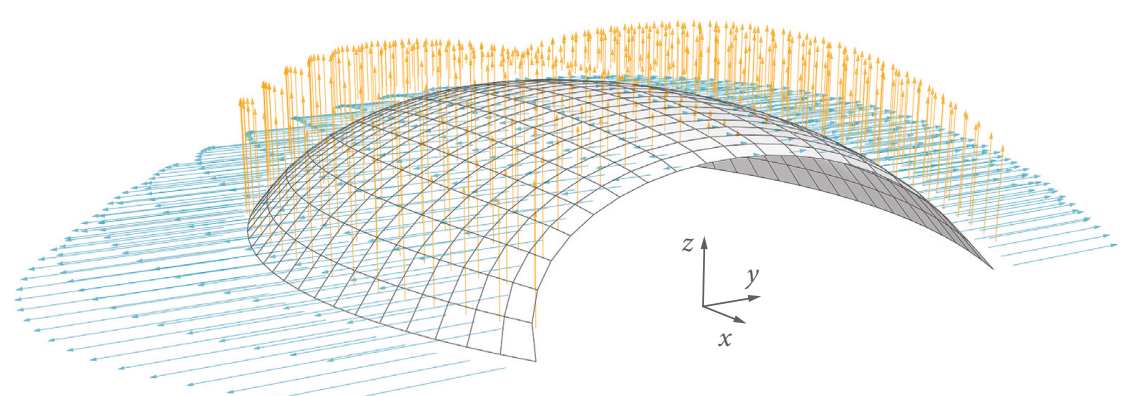

Figure 10: Applied forces representing shell self-weight, vertical earth load (orange), and horizontal earth pressure (blue).

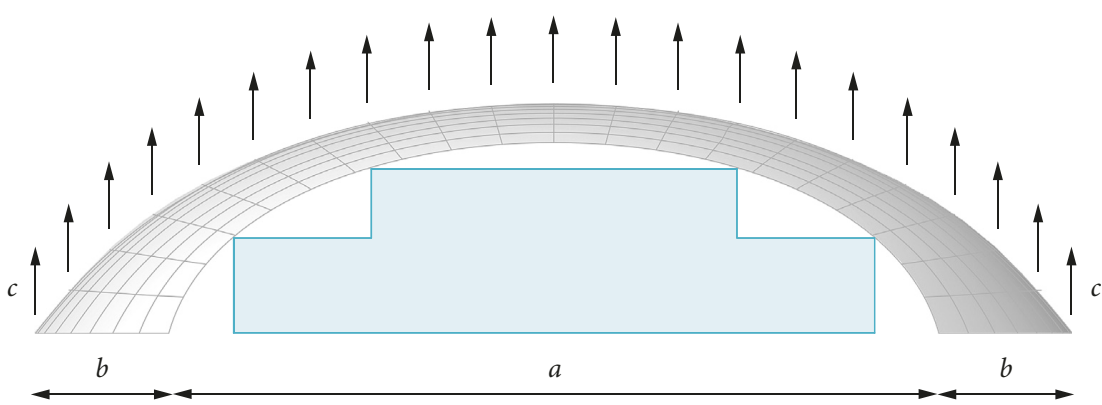

Figure 11: Structural gauge of the railway track to be enclosed.

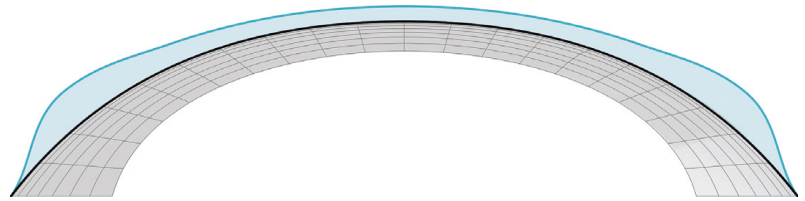

FiguRE 12: Curvature graph in the longitudinal direction.

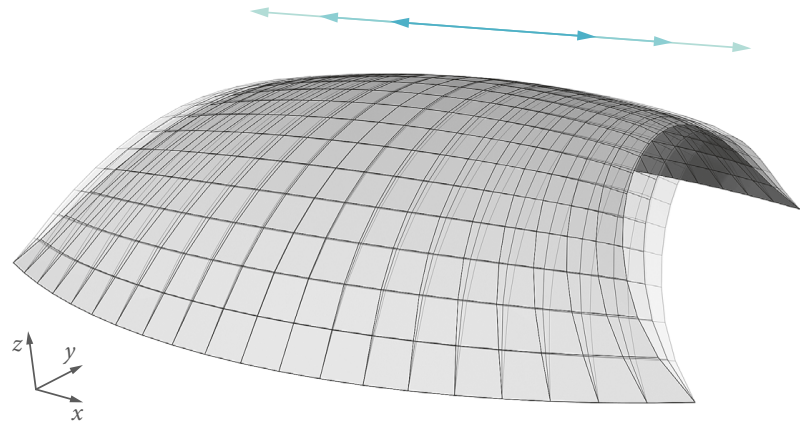

Figure 13: Modification of the width at the vertex of the shell.

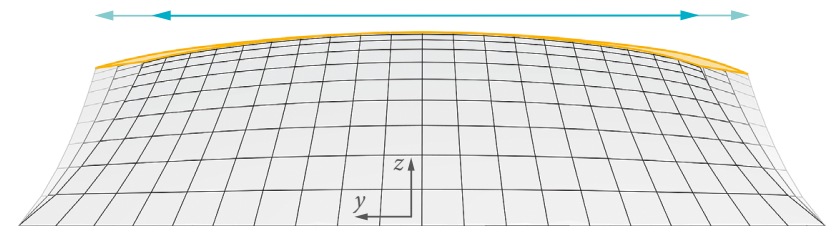

FIgURE 14: Increase of the width of the shell at its vertex.

different in the vertical and horizontal directions, still causes undesirable bending moments in the shell. To compensate that, an additional form adaption was performed according to the results of the finite-element calculations. The adaption is largest at the shell vertex and linearly decreases in the $x$ and $y$-directions (Figure 15). Due to these forces, the geometry is improved locally without violating any procedural requirements.

6.1.6. Generation of Input Data for Structural Analysis. In order to optimize the interface with the structural analysis software, the data are prepared automatically by the customized geometry optimization tool. The entire surface of the bridge was subdivided into different areas, so variable earth pressure could be applied over the height of the finiteelement model.

The magnitude of the load applied to the shell depends on two factors: the final terrain surface and the geometry of the shell. After completion of the form-finding process, the soil cover to be used for the subsequent calculations is determined automatically by the customized design tool. The tool measures the vertical distance from the shell to the final terrain surface at several predefined points (Figure 16). Subsequently, the distances are exported automatically and used to determine the vertical earth load and horizontal earth pressure, which are fed into the finite-element model for further static calculations.

6.1.7. Feedback Loop to Geometry. Several potential improvements were determined from the structural analysis carried out with finite-element calculations. The feedback loop illustrated in Figure 17 allows for an improvement of the shell geometry till an optimum is achieved which equally fulfills the various geometric, structural, economic, and aesthetic requirements. 


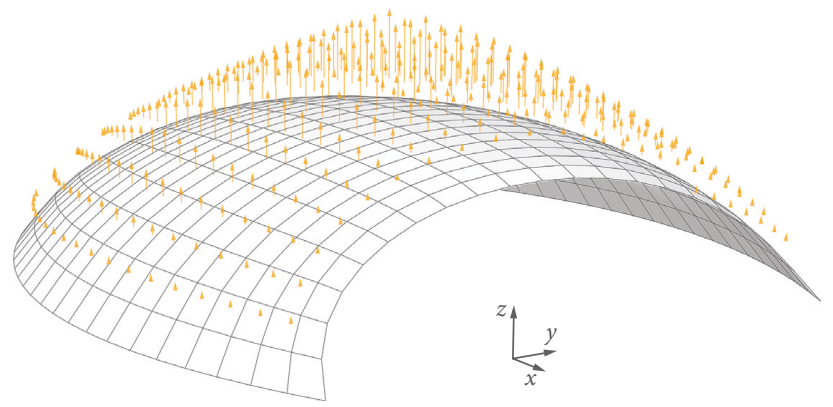

Figure 15: Illustration of the additional form adaption, fading two dimensionally from the shell vertex.

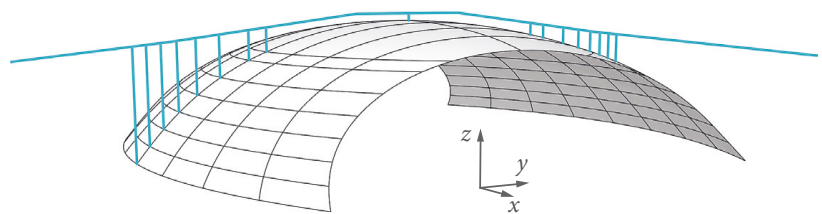

FIGURE 16: Illustration of the soil cover measurement.

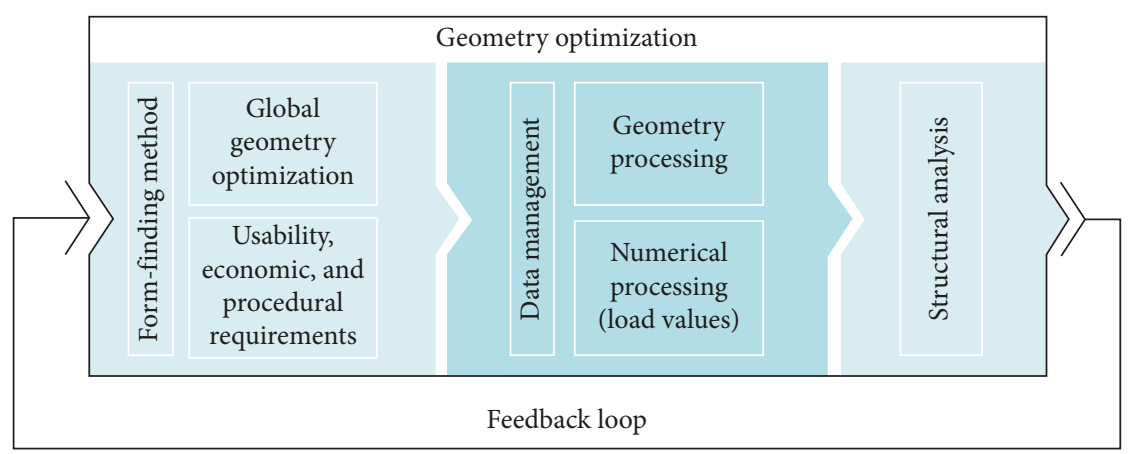

FIGURE 17: Diagram of the optimization process.

However, the geometry optimization is currently a semiautomated process. Using the findings of the structural calculation, the local modifiers are employed to slightly deform and hence improve the geometry. The updated geometry is transferred to the structural software and reanalyzed. This procedure is repeated until an optimum result is achieved.

6.1.8. Static Calculations. The basic static calculations within the geometry optimization are performed with the finiteelement program RFEM [24] using a linear-elastic material model. As the concrete remains uncracked under full load, this assumption yields accurate results. To reduce the number of influencing factors, a constant soil density is assumed for the optimization process. A wider range of load parameters is taken into consideration in the detailed static calculations that are carried out after the final geometry has been determined. As explained above, the geometry is split into vertical layers of one meter height and loaded with the vertical earth load and the horizontal earth pressure using so-called load blocks. The mean earth load is determined for each layer using the data automatically produced during the optimization, as explained above. The self-weight of the structure is automatically taken into consideration by the calculation program. The results of the static calculation are studied in detail and used as input data for the next optimization loop. After the final geometry has been found, a detailed structural analysis with additional variations of unfavorable loads is carried out.

6.2. Form Finding of the Reference Geometry Based on a Thrust-Network Approach (Alternative Step 1). Recent research on compression-only structures by Block et al. (e.g., $[19,25])$ has revealed numerous advantages of the socalled thrust-network approach, whose origin goes back to graphic statics. A mesh representing the desired shell must be found, with a compressive force acting on each mesh edge so that the resulting force in each mesh vertex balances the sum of loads acting on that vertex (self-weight, vertical earth loading, and horizontal earth pressure). In this project, a variant of the computational approach presented in [26], Guided Projection, was implemented because of its high 
computational efficiency, its ease of incorporating additional constraints, and the option of user interaction.

6.2.1. Overview. In our implementation, a manipulatable coarse control mesh is presented to users for interactive design. After user manipulation of the coarse mesh, a quaddominant mesh is generated based on a subdivision rule. This quad-dominant mesh represents the thrust network. Based on the quad-dominant mesh, axial forces are initialized in a least-squares sense to achieve a force balance with fixed preoptimized geometry. With the initialized forces and geometry as a starting point, the vertices are repositioned so as to achieve force equilibrium in the structure, in which only compressive forces are allowed to occur. During the computation, the loads imposed on the nodes are evaluated before each iteration. Additional constraints are imposed to ensure that the boundary vertices glide on a prescribed curve. Regularizers such as energy balance and tangential energy balance, which ensure the smoothness of the overall structure, are used with decaying weights. It is important to note that while the force-balance conditions are imposed only on the final permanent structure, the energy balance is imposed on the whole concrete shell including the parts which are later removed.

After user manipulation of the controlled mesh, the Guided Projection algorithm initializes the proper geometry and forces and then projects this initial guess towards a nearby valid solution point using fairness energies. The overall computation time is less than one second in a singlethread implementation. This performance is acceptable for interactive design. In the following sections, a detailed description of this approach is presented. First, the notation and initialization of the geometry are discussed. Next, force initialization and force-balance conditions are introduced. Then, other conditions and regularizers are described. Finally, the iterative scheme of Guided Projection is presented, and its computational efficiency is discussed.

As discussed above, a structure is statically sound if and only if there exists a thrust network within the physical shell which balances the loads. In practice, a quad-dominant mesh is used as the thrust network. Based on an initial control polygon, a subdivision scheme is applied to create a quad-dominant subdivision surface. The used rule is similar to the Catmull-Clark algorithm, except that triangles at the boundaries are allowed. Two parts are distinguished in the geometry: the permanent component that is kept after construction and the temporary part that will be removed, even if it is necessary for the inflation of the pneumatic formwork. Figure 18 illustrates the relationship between the control mesh and the initial geometry before optimization. In the following paragraphs, $(V, F, E),\left(V^{\prime}, F^{\prime}, E^{\prime}\right)$, and $(\bar{V}, \bar{F}, \bar{E})$ denote the sets of vertices, faces, and edges of the permanent structure, the temporary structure, and the whole inflatable structure, respectively. By definition, $V \cup V^{\prime}=\bar{V}$, $F \cup F^{\prime}=\bar{F}$, and $E \cup E^{\prime}=\bar{E}$. In Figure 19, we visualize all the edges $\bar{E}$ of the initial geometry and only the faces of the permanent component $F$. To achieve structural soundness, the permanent structure $(V, F, E)$ should be in static balance with the external loads without the part which is later removed. However, it is important for the inflation process that the entire dome, including the part to be removed, exhibits a smooth surface.

\subsubsection{Force-Balance Conditions and Force Initialization.} In order to create a concrete dome structure that satisfies force-balance requirements, it is necessary to consider the force-balance conditions. In general, the initial geometry does not admit a valid solution of balanced axial forces. To create a thrust network with balanced axial forces, it is necessary to simultaneously solve for vertex locations and the axial forces. To represent the axial forces, a scalar $w_{i j}$ is introduced for each edge connecting vertex $\mathbf{v}_{i}$ and vertex $\mathbf{v}_{j}$. The force density $w_{i j}$ is defined as the axial force divided by the edge length. The concept of force density simplifies force-balance conditions to bilinear equations. A vertex $\mathbf{v}_{i}$ is in force balance if and only if the resultant force of the adjacent edge cancels the load imposed on vertex $\mathbf{v}_{i}$ :

$$
\sum_{\{(i, j)\} \in E} w_{i j}\left(\mathbf{v}_{i}-\mathbf{v}_{j}\right)=-\mathbf{l}_{i}, \quad i \in V .
$$

As force-balance conditions are equations of vectors, there are three equations for each vertex. To initialize force densities, the vertex locations are kept fixed and force densities are solved in a least-squares sense. For form finding, the vertex locations $\mathbf{v}_{i}$ are treated as variables and solved simultaneously. The external loads $\mathbf{1}_{i}$ due to earth pressure and self-weight, which depend on the influence area of the vertex, are evaluated prior to each iteration.

6.2.3. Ensuring Compressive Forces Only. An edge in compression is associated with a positive force-density term. To ensure that there are no tensile forces, all force-density terms must be nonnegative: $w_{i j} \geq 0$. This condition can be ensured simply by introducing an auxiliary variable $u_{i j}$ for every edge and requiring that $w_{i j}=u_{i j}^{2}$ throughout the computation.

6.2.4. Boundary Alignment. As shown in Figure 19, all the boundary vertices $\partial \bar{V}$ of the shell must glide on a prescribed curve given by the initially subdivided surface. The $z$-coordinate of all the boundary vertices should be zero, i.e., the vertices should be located on the ground. The former requirement is ensured by

$$
\mathbf{v}_{i} \cdot \mathbf{n}_{i, k}=0, \quad i \in \partial \bar{V}, k \in\{1,2\},
$$

where vectors $\mathbf{n}_{i, 1}$ and $\mathbf{n}_{i, 2}$ are the two normal vectors, computed for an estimated tangent vector $\mathbf{t}_{i}$. As the tangent vector $\mathbf{t}_{i}$ is in the horizontal plane, the requirement that all the vertices should remain on the ground is automatically guaranteed by these equations. Figure 20 shows the mesh (the thrust network) resulting from the optimization process. 


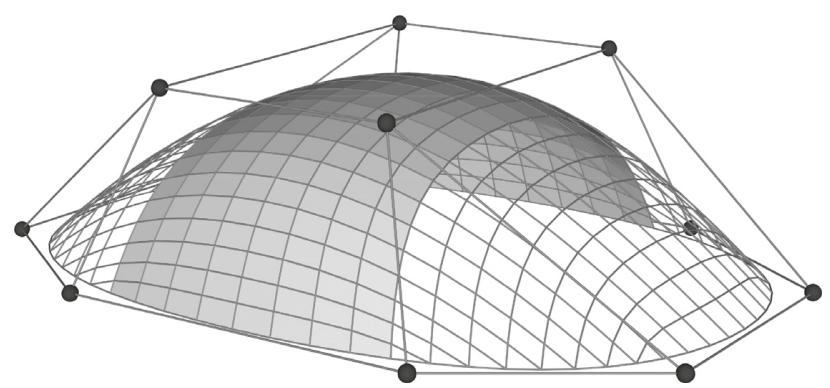

FIGURE 18: The control structure and the preoptimized geometry derived by a subdivision rule.

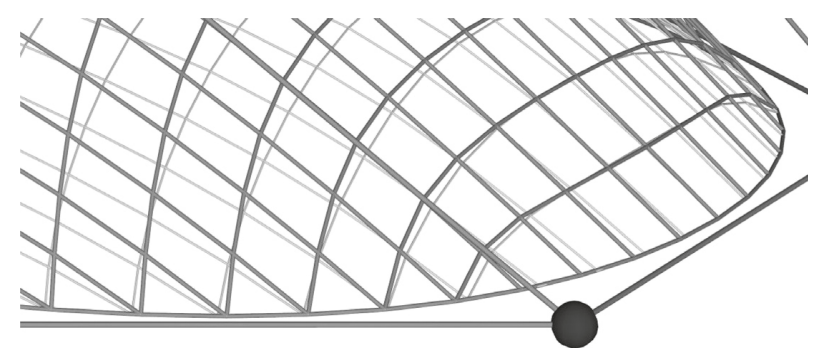

FIGURE 19: Boundary alignment of the resultant thrust network (lighter) based on the initial network (darker).

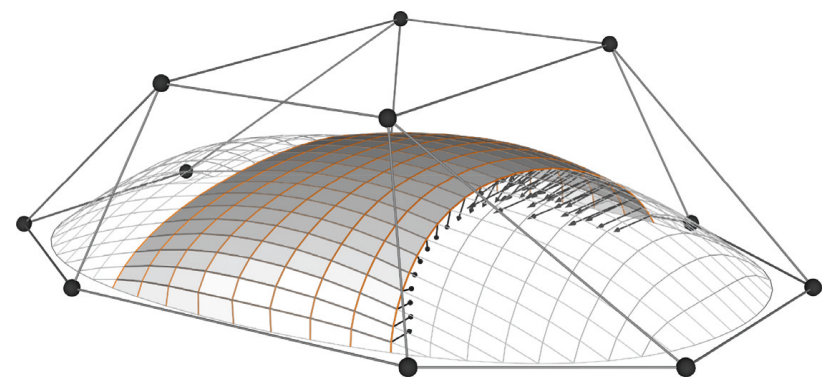

FIGURE 20: The mesh (the thrust network) resulting from form finding is in force balance with the imposed external loads.

6.2.5. Fairness. During the computation, the final concrete shell $(\bar{V}, \bar{F}, \bar{E})$ needs to be a smooth overall structure. In the discrete setting, smoothness is expressed by mesh fairness. For the quad-dominant mesh, the following polyline fairness is required for each mesh polyline:

$$
\mathbf{v}_{i} \approx \frac{\mathbf{v}_{i-1}+\mathbf{v}_{i+1}}{2}, \quad i \in \bar{V} .
$$

As the minimizers of such a requirement are polylines with points evenly distributed on a straight line, it would have the effect of oversmoothing and flattening. Therefore, these fairness expressions are applied with stronger weights to the tangential components, thus not acting against the normal curvature:

$$
\mathbf{t}_{i}^{k} \cdot \mathbf{v}_{i} \approx \mathbf{t}_{i}^{k} \cdot \frac{\mathbf{v}_{i-1}+\mathbf{v}_{i+1}}{2}, \quad i \in \bar{V}, k \in\{1,2\},
$$

where the two mutually orthogonal unit tangent vectors $\mathbf{t}_{i}^{1}$ and $\mathbf{t}_{i}^{2}$ are computed from the vertex normal $\mathbf{n}_{i}$ prior to each iteration.
6.2.6. Iterative Procedure and Computational Efficiency. Based on the formulation above, the system to be solved is a collection of quadratic equations that include the forcebalance conditions and the auxiliary conditions to ensure that all the axial forces are nonnegative. This collection of quadratic equations, $\varphi_{i}$, is denoted in the general form as

$$
\varphi_{i}(\mathbf{x})=\frac{1}{2} \mathbf{x}^{T} H_{i} \mathbf{x}+\mathbf{b}_{i}^{T} \mathbf{x}+c_{i}=0, \quad i=1, \ldots, N
$$

where the vector, $\mathbf{x}$, encodes all the variables to be determined. To solve this collection of quadratic equations, a Newton-like procedure is applied. Starting from the variables $\mathbf{x}_{n}$ at the $n$th iteration, the quadratic equations are linearized by the Taylor expansion:

$$
\varphi_{i}(\mathbf{x}) \approx \varphi_{i}\left(\mathbf{x}_{n}\right)+\nabla \varphi_{i}\left(\mathbf{x}_{n}\right)^{T}\left(\mathbf{x}-\mathbf{x}_{n}\right)=0, \quad i=1, \ldots, N .
$$

The linearized equations are solved in a least-squares sense together with the aesthetic regularizers, i.e., the equations expressing fairness mentioned above: 


$$
\left\|\varepsilon_{h}(H \mathbf{x}-\mathbf{r})\right\|^{2}+\left\|\varepsilon_{s}(K \mathbf{x}-\mathbf{s})\right\|^{2}+\left\|\delta\left(\mathbf{x}-\mathbf{x}_{n}\right)\right\|^{2} \longrightarrow \min .
$$

The last term, $\left\|\delta\left(\mathbf{x}-\mathbf{x}_{n}\right)\right\|^{2}$, is a regularizer for numerical stability, preventing the solution from drifting away from a reasonable initial guess. In the presented implementation, a fixed number of ten iterations is used for form finding. The relative coefficient, $\varepsilon_{h}$, set to 1.0 , is for hard constraints (force-balance conditions, boundary alignment, and compressive force requirements). The numerical stability coefficient, $\delta$, is set to 0.0001 . The relative coefficient $\varepsilon_{s}$ for fairness terms is set to 0.1 , with a decaying coefficient of 0.9 repetitively multiplied for the first five iterations. After the first five iterations, $\varepsilon_{s}$ is set to zero. Afterwards, only the hard constraints are used in the computation; the fairness terms are left out. Adaptively changing the coefficient, $\varepsilon_{s}$ ensures that the force-balance conditions are strictly satisfied.

Regarding computational efficiency, the overall computation time is less than one second, even for a singlethreaded nonoptimized implementation. The achieved force-balance condition is in the order of $10^{-6}$, measured by the average per-vertex ratio between the magnitude of force residual and external load. Further discussion about the computational efficiency of the iterative procedure, Guided Projection, can be found in [26].

6.3. Completion of the Cupola (Step 2). Up to this point, the focus of the process has been on the reference geometry of the final shape. However, a full cupola must be erected in this construction method. The completion of the reference geometry requires multiple steps. First, the two bottom edges are connected to form a closed curve which is as continuous as possible. Discontinuities must be avoided, as they would cause stress peaks in the thin concrete structure during the transformation process. Secondly, the longitudinal section curve of the bridge is extended to the intersection of the bottom-edge curve with the $x$-axis (Figure 21).

Using the generated curves, the existing reference geometry is completed to form a full cupola, which serves as the basis for the discretization process.

6.4. Discretization and Unrolling of the Reference Geometry (Steps 3 and 4). The fact that each segment of the flat concrete plate can only be bent in one direction requires discretization of the double-curved cupola into a sequence of developable surface strips. As described in [28], such a developable strip model (D-strip model) is the limit of a quad mesh with planar faces (PQ mesh) in a refinement procedure where only the rows (or the columns) of the mesh are refined. The discretization process was realized using the software "EvoluteTools" [29], a plugin for Rhinoceros.

First, a coarse mesh is modeled that forms a very rough discrete representation of the reference geometry and determines the number of developable segments. These segments represent the basis of a grid in which the rows are refined gradually. After each refinement, the resulting mesh is optimized. EvoluteTools allows various optimization criteria to be set and weighted. In the presented case, the planarity of each mesh face, expressed through the shortest distance between face diagonals, is the main criterion. The implemented algorithm tries to minimize the sum of the squared distances. If all distances are zero, the strips are perfectly developable. In order to keep the optimized geometry as close as possible to the reference geometry, several other criteria are considered. The criterion "surface closeness" tries to keep the position of each mesh vertex as close to the reference geometry as possible, while the criterion "curve closeness" seeks to minimize the distance of the boundary vertices to the nearest boundary of the reference geometry. In most cases, the optimization criteria are in conflict with each other, which means that not all of them are fulfilled perfectly in the result. With the help of the assigned weights, the user is able to determine the importance of the individual criteria and thus influence the outcome of the optimization (Figure 22). For details on the optimization, which is a nonlinear least-squares problem and solved with a properly regularized Gauss-Newton algorithm, refer to $[27,28]$.

The refinement process is concluded when the single strips have a Gaussian curvature of zero. This allows for them to be unrolled into a planar representation without experiencing any distortion (step 4). The resulting shape forms the basis for the simple, planar formwork (Figure 23).

It should be pointed out that there is an infinite number of D-strip models which approximate a given surface. In our case in particular, with the surface to be approximated being convex, many of these $\mathrm{D}$-strip models may be aesthetically pleasing solutions from a purely geometric perspective. Two promising options among the range of possible solutions are (i) an attempt could be made to achieve rulings (straight line segments) of the developable strips that are as orthogonal as possible to the strip boundaries. This option yields a so-called principal strip model and can be achieved with EvoluteTools, choosing as additional constraints for the mesh optimization condition that mesh faces have a circumcircle or mesh vertices be conical [29]. The solution shown in Figure 22 is close to a principal strip model. Another interesting option would be to aim at obtaining unrolled strips whose boundaries are as straight as possible. In this case, the developable strips follow shortest paths (geodesics) on the cupola surface. This approach requires another term in the optimization and yields a so-called geodesic strip model [27]. Various geodesic strip models were computed for the data set of the investigated bridge, but they were not used due to an uneven distribution of strip widths or other features which may cause problems during the inflation process.

\section{Discussion}

The presented paper describes a practically oriented approach for the optimization of the shape of shell bridges built with PFHC. A hanging model simulated with a particle-spring system is used, as explained in Section 6.1. Additionally, a new optimization approach is presented in Section 6.2. The following boundary conditions were assumed as given: 

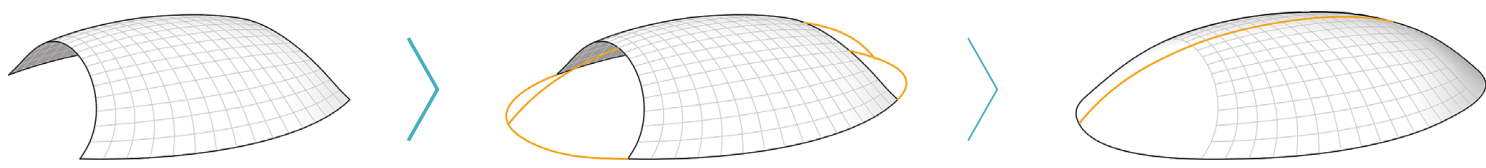

Figure 21: Completion of the full cupola.
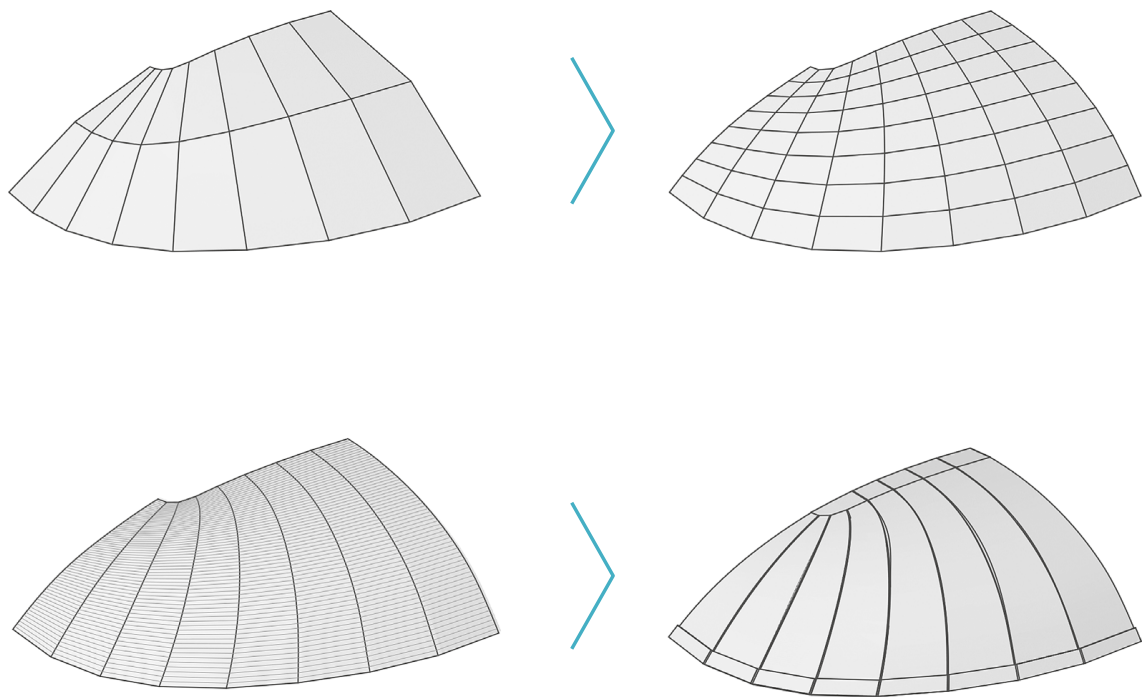

FIgURE 22: Discretization process from the coarse mesh to a developable strip model.

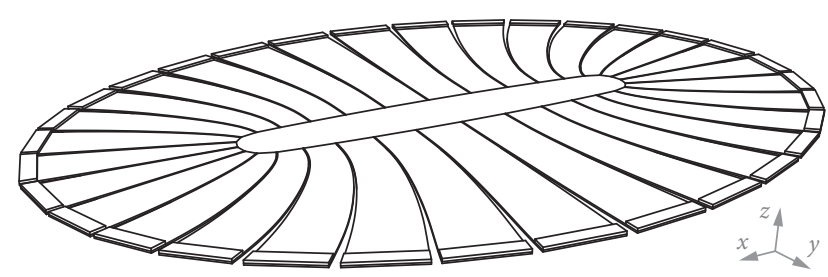

FIGURE 23: Flat planar formwork, ready to be lifted.

(i) A constant thickness of the shell

(ii) Uniform material properties over the entire shell surface

(iii) A linear relationship between the vertical/horizontal earth pressure and the soil cover

(iv) A smooth surface and continuous change of curvature (no ribs or folds)

(v) No inherent rigidity of the dynamic particle system

7.1. Improvement of Material Utilization and Shell Stiffness (Buckling Load). The assumptions listed above show further potential for improvement of the optimization process. For example, the thickness of the shell could be optimized to vary over the surface to minimize material where possible.

Unfortunately, various influencing factors lead to unintended bending moments within the already optimized structure. These include (i) Unexpected asymmetric loads due to the shell selfweight (e.g., because of varying shell thicknesses)

(ii) Unexpected asymmetric live loads (e.g., earth load)

(iii) Shrinkage and creep

Although mainly normal compressive forces occur in the designed structure, these unfavorable bending moments have to be managed by installing additional reinforcement. In another possible approach, the stiffness of the shell could be improved by structural optimization of the shape where necessary. The influence of initial geometric imperfections on the buckling load of single and double curvature concrete shells is explained in [30]. Basically, the thin shell must have a constant thickness, so that PFHC can be used, but the additional concrete layer can have almost any shape. For example, some kind of ribs could be formed. Another possibility would be to introduce graded concrete as explained in [31] to create concrete ribs or have concrete areas with higher or lower densities. This allows a reduction of the self-weight and requires less concrete. A detailed analysis of the buckling behavior of the application of the construction method for a shell bridge used as deer pass resulted in a buckling load factor of 22 (for the special project currently built in Austria). The buckling behavior for this special application can therefore be classified as uncritical. Reasons are the comparatively high thickness of $0.45 \mathrm{~m}$ and the favorable mode of action of the earth loads, since the resistance of the earth is increasing by a movement from the concrete support structure (passive earth pressure) and decreasing by a movement from the concrete away from the earth (active earth pressure). 
7.2. Increase of the Level of Automation within the Geometry Optimization. As described in Section 6.1.7, the findings from the structural analysis are transferred manually to the modification process of the parameters of the geometric optimization. Further automatization of this process would simplify the optimization and accelerate the process significantly.

Linking the currently separated discretization process directly to the geometry optimization would be another possibility for further improvement.

The production of the formwork for the flat plate (wedges and edge formwork) requires very high accuracy, since the accuracy of the final shape is directly linked to the accuracy of the initially flat plate. To ensure a true-to-size production, it is recommended to use the computer numerically controlled mills. The generation of the output files can therefore also be included in the automated optimization process.

\section{Conclusions}

The presented paper describes the design of the shape concrete shell bridge constructed with the newly invented construction method Pneumatic Forming of Hardened Concrete (PFHC). The optimization process is performed in three steps: form finding of the reference geometry, completion of the cupola, and discretization and unrolling of the geometry.

The concept of reversed engineering allows the main task-the optimization process of the final form with respect to the acting loads and the construction method-to be tackled first. First, optimization by using a particle-spring system is described. Furthermore, a new optimization approach using a thrust-network approach is presented. The determined form is completed to form a cupola, discretized to a flat plate, and subsequently unrolled. During the optimization process, all requirements could be taken into account with a weighting in relation to their importance.

It is shown that the high complexity of a practical design process can be simplified and semiautomated by using specialized CAD and structural analysis software. The data of the free-form surface can be processed for further planning and allows exact production at the building site.

Numerical form-finding methods provide an efficient support tool to take into account many different physical properties within the structural optimization process. Even so, the final shape is influenced by a large number of different boundary conditions, leading in most of the cases to multiple solutions. Thus, currently, it depends on the engineer's evaluation and interpretation of the different influencing factors to determine an appropriate shape. In the presented project, close cooperation of all engineers involved, and an interdisciplinary way of thinking allowed for steady improvement of the optimization concept and led to a successful solution for the sophisticated task.

\section{Data Availability}

The data used to support the findings of this study are included within the article.

\section{Conflicts of Interest}

The authors declare that they have no conflicts of interest.

\section{Acknowledgments}

The presented research was performed accompanying the design process of a shell bridge in Austria. The authors would like to thank ÖBB-Infrastruktur AG for the great cooperation. The publication of the present paper was supported by BOKU Vienna Open Access Publishing Fund.

\section{References}

[1] B. Kromoser and P. Huber, "Pneumatic formwork systems in structural engineering," Advances in Materials Science and Engineering, vol. 2016, Article ID 4724036, 13 pages, 2016.

[2] B. Kromoser and J. Kollegger, "Herstellung von Schalentragwerken aus Beton mit der "Pneumatic Wedge Method"," Beton-und Stahlbetonbau, vol. 109, no. 8, pp. 557-565, 2014.

[3] B. Kromoser and J. Kollegger, "Application areas for pneumatic forming of hardened concrete," Journal of the International Association for Shell and Spatial Structures, vol. 56, no. 3, pp. 187-198, 2015.

[4] B. Kromoser and J. Kollegger, "Pneumatic forming of hardened concrete-building shells in the 21st century," Structural Concrete, vol. 16, no. 2, pp. 161-171, 2015.

[5] B. Kromoser and J. Kollegger, "Aktives Verformen von ausgehärteten Betonelementen zur Herstellung von räumlich gekrümmten Betonflächen," Beton-und Stahlbetonbau, vol. 112, no. 2, pp. 106-115, 2017.

[6] W. Sobek, Auf pneumatisch gestützten Schalungen hergestellte Betonschalen, Ph.D. dissertation, University of Stuttgart, Stuttgart, Germany, 1987.

[7] A. Tomás and P. Martí, "Shape and size optimisation of concrete shells," Engineering Structures, vol. 32, no. 6, pp. 1650-1658, 2010.

[8] K.-U. Bletzinger and E. Ramm, "Structural optimization and form finding of light weight structures," Computers \& Structures, vol. 79, no. 22-25, pp. 2053-2062, 2001.

[9] S. I. Robles and N. F. Ortega, "Study of volumetric displacements of shells," Journal of the International Association for Shell and Spatial Structures, vol. 42, no. 3, pp. 139-147, 2001.

[10] M. Barnes, "Form and stress engineering of tension structures," Structural Engineering Review, vol. 6, no. 3, 1994.

[11] H. Isler, "Concrete shells derived from experimental shapes," Structural Engineering International, vol. 4, no. 3, pp. 142-147, 1994.

[12] K. Linkwitz and H.-J. Schek, "Einige Bemerkungen zur Berechnung von vorgespannten Seilnetzkonstruktionen," Ingenieur-Archiv, vol. 40, no. 3, pp. 145-158, 1971.

[13] K.-U. Bletzinger, Form Finding and Optimization of Membranes and Minimal Surfaces, Institut for Structural Analysis, University of Karlsruhe, Karlsruhe, Germany, 1998.

[14] E. Haug, "Finite element analysis of nonlinear membrane structures," in Proceedings of the IASS Pacific Symposium on Tension Structures and Space Frames, Tokyo, Japan, October 1971.

[15] T. Suzuki, "Shape analysis of minimal surface by the finite element method," in Proceedings of the International IASS Symposium on Spatial Structures at the Turn of the Millenium, pp. 103-110, Copenhagen, Denmark, September 1991. 
[16] D. Veenendaal and P. Block, "An overview and comparison of structural form finding methods for general networks," International Journal of Solids and Structures, vol. 49, no. 26, pp. 3741-3753, 2012.

[17] H. Pottmann, A. Asperl, M. Hofer, and A. Kilian, Architectural Geometry, Vol. 724, Bentley Institute Press Exton, Exton, PA, USA, 2007.

[18] B. Kromoser, J. Kollegger, H. Kari, K. Gradenegger, and M. Ganster, "Ein innovatives Betonschalenbauverfahren in Anwendung: Herstellung der Wildbrücke AM2 mit PFHC," Beton-und Stahlbetonbau, vol. 113, no. 3, pp. 222-232, 2018.

[19] S. Adriaenssens, P. Block, D. Veenendaal, and C. Williams, Shell Structures for Architecture: Form Finding and Optimization, Routledge, Abingdon, UK, 2014.

[20] "Kangaroo-Grasshopper," October 2018, https:// www.grasshopper3d.com/group/kangaroo.

[21] "Rhino 6 für Windows," September 2018, https:// www.rhino3d.com/de/.

[22] S. D. created this N. Network, "Grasshopper," September 2018, https://www.grasshopper3d.com/.

[23] B. Kromoser and J. Kollegger, "Entwurf, Geometrieoptimierung und Bemessung der Wildbrücke AM2 hergestellt mit PFHC," Beton-und Stahlbetonbau, vol. 113, no. 2, pp. 88-95, 2018.

[24] "RFEM: Structural FEM Analysis \& Design Software | Dlubal Software," October 2018, https://www.dlubal.com/en/ products/rfem-fea-software/what-is-rfem.

[25] P. Block, Thrust network analysis: exploring three-dimensional equilibrium, Ph.D. dissertation, Massachusetts Institute of Technology, Cambridge, MA, USA, 2009.

[26] C. Tang, X. Sun, A. Gomes, J. Wallner, and H. Pottmann, "Form-finding with Polyhedral meshes made simple," ACM Transactions on Graphics, vol. 33, no. 4, pp. 1-9, 2014.

[27] H. Pottmann, A. Schiftner, P. Bo et al., "Freeform surfaces from single curved panels," ACM Transactions on Graphics, vol. 27 , no. 3, p. 76, 2008.

[28] Y. Liu, H. Pottmann, J. Wallner, Y.-L. Yang, and W. Wang, "Geometric modeling with conical meshes and developable surfaces," ACM Transactions on Graphics, vol. 25, no. 3, pp. 681-689, 2006.

[29] "Evolute | the Geometry Experts," October 2018, http:// www.evolute.at/.

[30] A. Tomás and J. P. Tovar, "The influence of initial geometric imperfections on the buckling load of single and double curvature concrete shells," Computers \& Structures, vol. 96-97, pp. 34-45, 2012.

[31] M. Herrmann and W. Sobek, "Functionally graded concrete: numerical design methods and experimental tests of massoptimized structural components," Structural Concrete, vol. 18, no. 1, pp. 54-66, 2017. 


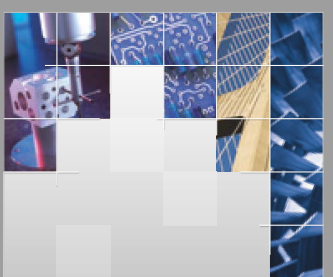

\section{Enfincering}
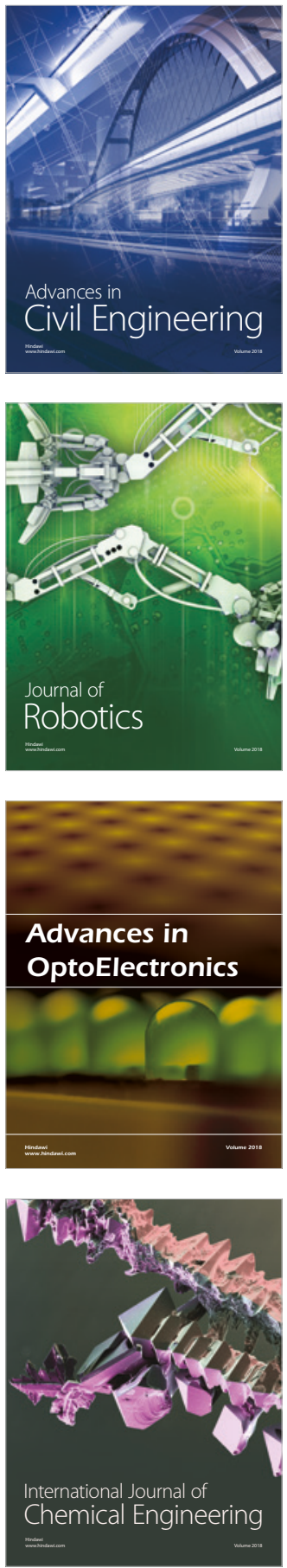

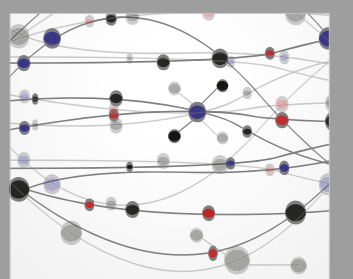

\section{Rotating \\ Machinery}

The Scientific World Journal

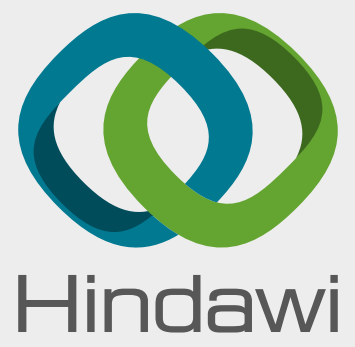

Submit your manuscripts at

www.hindawi.com
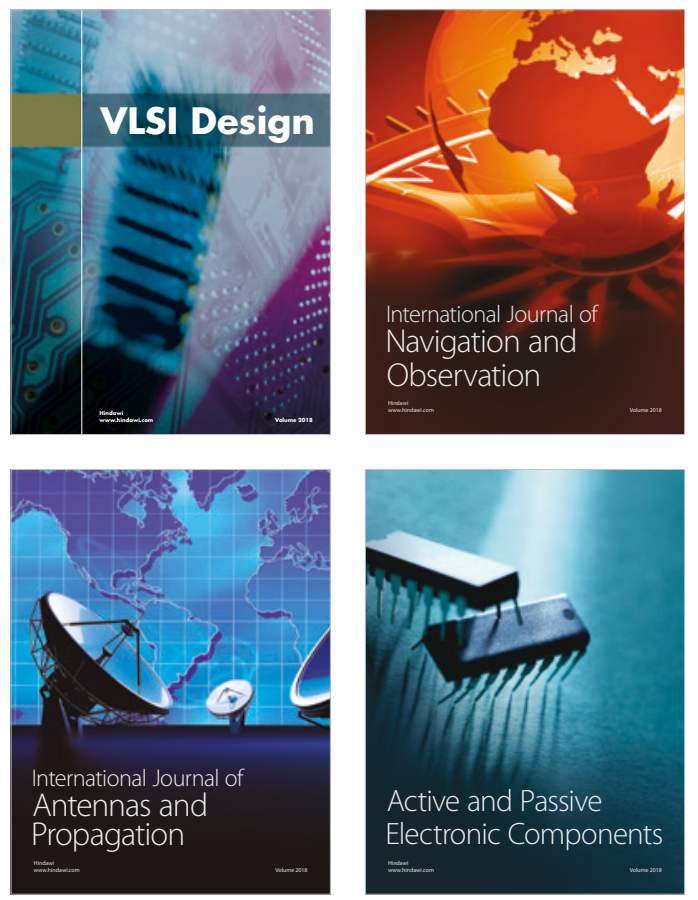
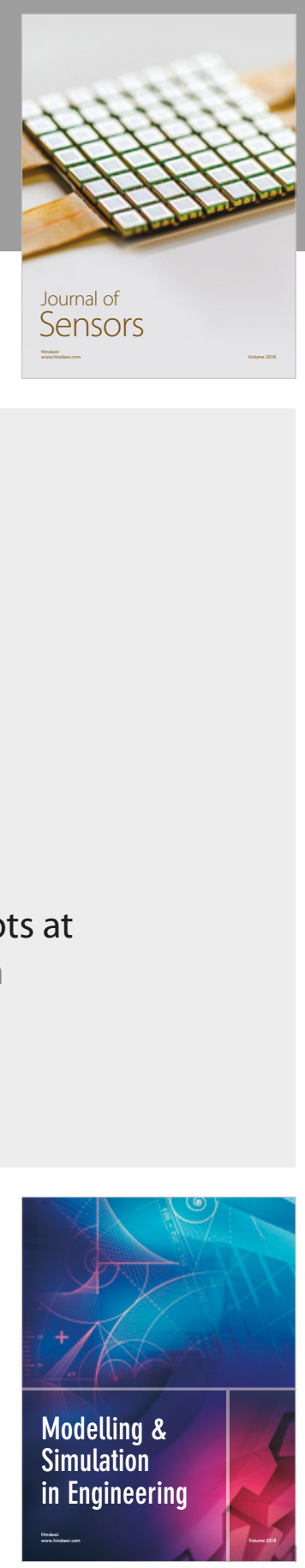

\section{Advances \\ Multimedia}
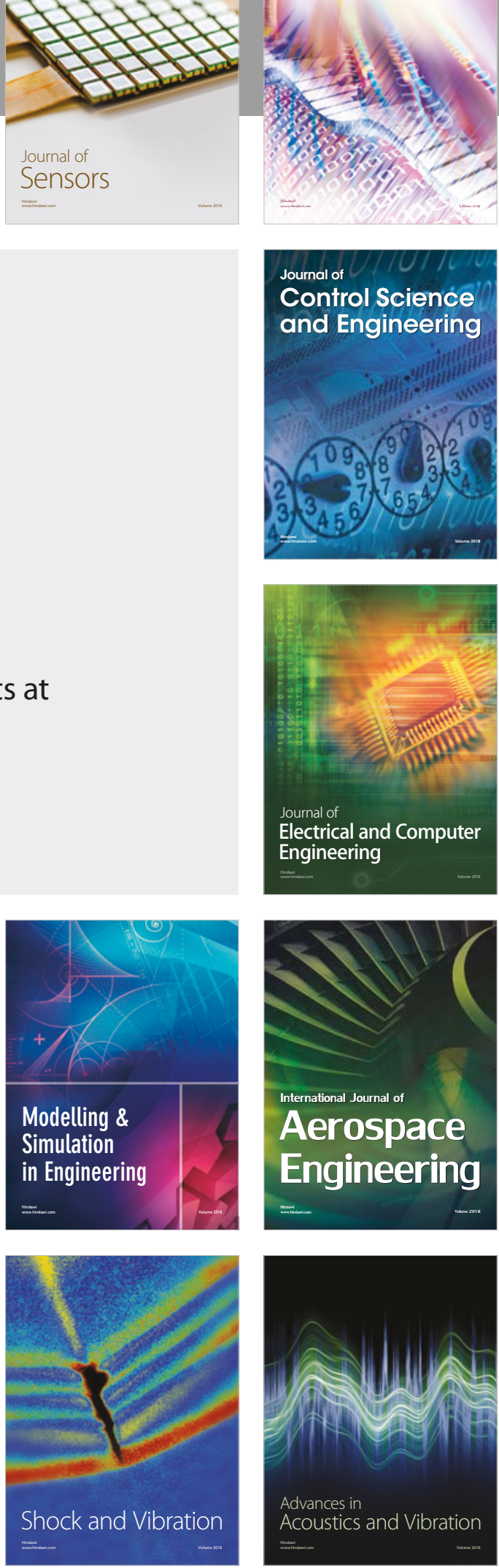